\title{
A Global Energy Function for the Alignment of Serially Acquired Slices
}

\author{
Stelios Krinidis, Christophoros Nikou, Associate Member, IEEE, and Ioannis Pitas, Senior Member, IEEE
}

\begin{abstract}
An accurate, computationally efficient, and fully automated algorithm for the alignment of two-dimensional (2-D) serially acquired sections forming a three-dimensional (3-D) volume is presented. The approach relies on the optimization of a global energy function, based on the object shape, measuring the similarity between a slice and its neighborhood in the 3-D volume. Slice similarity is computed using the distance transform measure in both directions. No particular direction is privileged in the method avoiding global offsets, biases in the estimation and error propagation. The method was evaluated on real images [medical, biological, and other computerized tomography (CT) scanned 3-D data] and the experimental results demonstrated its accuracy as reconstuction errors are less than one degree in rotation and less than one pixel in translation.
\end{abstract}

Index Terms-Deterministic optimization, image registration, misalignment, nonoverlapping structures, pixel similarity measure, registration error, serially acquired images.

\section{INTRODUCTION}

$\mathbf{T}$ HREE-DIMENSIONAL (3-D) reconstruction of medical images [tissue sections, computerized tomography (CT) and autoradiographic slices] is now an integral part of biomedical research. In biomedicine, 3-D data are acquired by a multitude of imaging devices [magnetic resonance imaging (MRI), CT, 3-D microscopy, etc.]. In most cases, 3-D images are represented as a sequence of two-dimensional (2-D) parallel image slices. Depending on the acquisition method, the 2-D slices can be aligned or not. MRI images, for example, are aligned, whereas slices obtained through physical sectioning, e.g., biological tissue slices obtained using a microtome, are not. Usually alignment of such images involves only rotation and translation compensation. Therefore, reconstruction of such data sets into 3-D volumes, via the registrations of 2-D sections, has gained an increasing importance. The registration of multiple slices is very important for the correct 3-D visualization and morphometric analysis (e.g., surface and volume representation) of the structures of interest. Several alignment algorithms have been proposed in that framework. A review of general medical image registration methods is presented in [1], [2].

The 3-D alignment (reconstruction from 2-D images) methods may be classified in the following categories: fiducial marker-based methods [3], feature-based methods using con-

Manuscript received June 5, 2001; revised April 23, 2002 and August 30, 2002. This work was supported by the Greek General Secretariat of Research and Technology in the framework of PENED.

The authors are with the Department of Informatics Aristotle University of Thessaloniki, 54006 Thessaloniki, Greece (e-mail: pitas@zeus.csd.auth.gr).

Digital Object Identifier 10.1109/TITB.2003.811866 tours [4], crest lines or characteristic points extracted from the images [5], and gray-level-based registration techniques using the intensities of the whole image [6]-[8]. Most of the above mentioned techniques do not simultaneously consider the two major difficulties involved in medical and CT scanned data registration.

At first, consecutive slices may differ significantly due to distortions, discontinuities in anatomical structures, cuts, and tears. These effects are more pronounced when distant slices are involved in the registration. From this point of view, a registration method must be robust to missing data or outliers [8].

Besides, registering the slices sequentially (the second with respect to the first, the third with respect to the second, etc.) sometimes leads to misregistration. If an error occurs in the registration of a slice with respect to the preceding slice, this error will propagate through the entire volume. Furthermore, if the number of slices to be registered is large, a global offset of the volume may be observed, due to error accumulation [6].

In this paper, a solution to the above mentioned shortcomings is presented. A global energy function having as variables the rigid transformation parameters (2-D translation and rotation) of a given slice with respect to a local symmetric neighborhood is proposed. Global energy functions are a powerful tool in computer vision applications but they have not yet been considered for the registration of serially acquired slices. Our approach was inspired by the technique presented in [9], which consists in minimizing a global energy function with the iterative closest point algorithm [10] to register multiple, partially overlapping views of a 3-D structure. The global energy function implemented in our approach is associated with a pixel similarity metric based on the Euclidean distance transform [11].

The remainder of the paper is organized as follows. The global energy function formulation and the associated registration algorithm is presented in Section II, experimental results are presented in Section III and conclusions are drawn in Section IV.

\section{Global ENERGy FunCtion Formulation}

Before presenting the alignment method, the notations used in our formulation are introduced. A set of 2-D serially acquired slices is represented by

$$
V=\left\{I_{i}, i=1 \ldots N\right\}
$$

where $I_{i}$ is a slice (a 2-D image) and $N$ denotes the total number of slices. A pixel of a 2-D slice is represented by $\boldsymbol{p}=(x, y)^{T}$, so that $I_{i}(\boldsymbol{p})$ corresponds to the gray level (intensity) of pixel $p$ 
of slice $i . N_{x}$ and $N_{y}$ designate the number of pixels of each slice in the horizontal and vertical direction, respectively.

Standard 2-D rigid alignment consists of estimating the rigid transformation parameters (translation $t_{x}, t_{y}$ and rotation by angle $\theta$ ) that have to be applied to the image to be aligned (floating image) in order to match a reference image. In the approach proposed here, the alignment of the 2-D sections within the 3-D volume is considered globally, by minimizing an energy function $E(\cdot)$, which expresses the similarity between the 2-D sections

$$
E(\boldsymbol{\Theta})=\sum_{i=1}^{N} \sum_{j=1}^{N} \sum_{p=1}^{N_{x} \times N_{y}} f\left(I_{i}\left(\boldsymbol{T}_{\boldsymbol{\Theta}_{\boldsymbol{i}}}(\boldsymbol{p})\right), I_{j}\left(\boldsymbol{T}_{\boldsymbol{\Theta}_{\boldsymbol{j}}}(\boldsymbol{p})\right)\right)
$$

where $f(\cdot)$ is a similarity metric, $I_{k}$ denotes slice $k$ and $\boldsymbol{T}_{\Theta_{\boldsymbol{k}}}$ designates a rigid transformation with parameters $\Theta_{\boldsymbol{k}}=\left\{t_{x}^{k}, t_{y}^{k}, \theta^{k}\right\}$. Equation (2) indicates that, for a given set of rigid transformation parameters $\boldsymbol{T}_{\Theta_{i}}$, applied to the slice to be aligned $I_{i}$, the similarity between the transformed slice $I_{i}\left(\boldsymbol{T}_{\Theta_{i}}(p)\right)$ and all of the other already transformed slices $I_{j}\left(\boldsymbol{T}_{\Theta_{j}}(p)\right)$ in the volume is accumulated in the energy function. Assuming that function $f(\cdot)$ is symmetric

$$
\begin{aligned}
f\left(I_{i}\left(\boldsymbol{T}_{\Theta_{\boldsymbol{i}}}(\boldsymbol{p})\right), I_{j}\left(\boldsymbol{T}_{\Theta_{\boldsymbol{j}}}(\boldsymbol{p})\right)\right) \\
=f\left(I_{j}\left(\boldsymbol{T}_{\Theta_{\boldsymbol{j}}}(\boldsymbol{p})\right), I_{i}\left(\boldsymbol{T}_{\Theta_{\boldsymbol{i}}}(\boldsymbol{p})\right)\right)
\end{aligned}
$$

which is the case for the pixel similarity functions considered here (2), leads to the following global minimization problem:

$$
\begin{aligned}
& \hat{\boldsymbol{\Theta}}= \arg \min _{\Theta} E(\boldsymbol{\Theta}) \\
&=\arg \min _{\Theta} \sum_{i=1}^{N} \sum_{\substack{j=1 \\
j<i}}^{N} \sum_{p=1}^{N_{x} \times N_{y}} \\
& \cdot f\left(I_{i}\left(\boldsymbol{T}_{\boldsymbol{\Theta}_{\boldsymbol{i}}}(\boldsymbol{p})\right), I_{j}\left(\boldsymbol{T}_{\boldsymbol{\Theta}_{\boldsymbol{j}}}(\boldsymbol{p})\right)\right) .
\end{aligned}
$$

Without additional constraints, the optimization problem (4) has clearly an infinite number of solutions (if the set of rigid transformations $\left\{\boldsymbol{T}_{\hat{\Theta}_{1}}, \boldsymbol{T}_{\hat{\Theta}_{2}}, \ldots, T_{\hat{\Theta}_{\boldsymbol{N}}}\right\}$ is a solution, the same holds true for $\left\{\boldsymbol{T}_{\hat{\Theta}_{1}} \circ \boldsymbol{T}_{\Delta}, \boldsymbol{T}_{\hat{\Theta}_{2}} \circ \boldsymbol{T}_{\Delta}, \ldots, \boldsymbol{T}_{\hat{\Theta}_{\mathrm{\Theta}}} \circ \boldsymbol{T}_{\Delta}\right\}$, where $\boldsymbol{T}_{\boldsymbol{\Delta}}$ is an arbitrary 2-D rigid transformation). To remove this ambiguity, the transformation $\mathbf{T}_{\hat{\Theta}_{1}}$ applied to an arbitrary chosen slice $k$ is constrained to the identity transformation (we have chosen $k=1$ in our implementation). As a result, there are $3(N-1)$ parameters to estimate. It is common sense that distant slices present very little similarity due to anatomy and it would be more appropriate to measure the energy function only for slices presenting at least some similarities. Therefore, the support region of function $f(\cdot)$ has been limited to a neighborhood of radius $R$ centered at each slice and set

$$
f\left(I_{i}\left(\boldsymbol{T}_{\Theta_{\boldsymbol{i}}}(\boldsymbol{p})\right), I_{j}\left(\boldsymbol{T}_{\boldsymbol{\Theta}_{\boldsymbol{j}}}(\boldsymbol{p})\right)\right)=0, \quad \forall|i-j|>R .
$$

Thus, the following alignment algorithm is associated with the energy function (4): do until convergence:

declare all slices unvisited.

do until all slices are declared vis-

ited:

randomly choose an unvisited slice

$I_{i} \in V$.

update the rigid transformation param-

eters $\boldsymbol{T}_{\Theta_{\boldsymbol{i}}}$ bringing into alignment slice $I_{i}$ with the other slices in the neighborhood of $i$, by minimization of the following local energy function:

$$
\begin{aligned}
E_{i}\left(\boldsymbol{\Theta}_{\boldsymbol{i}}\right)=\sum_{i=1}^{N} \sum_{\substack{j=1 \\
|i-j| \leq R}}^{N} \sum_{p=1}^{N_{x} \times N_{y}} \\
\cdot f\left(I_{i}\left(\boldsymbol{T}_{\boldsymbol{\Theta}_{\boldsymbol{i}}}(\boldsymbol{p})\right), I_{j}\left(\boldsymbol{T}_{\boldsymbol{\Theta}_{\boldsymbol{j}}}(\boldsymbol{p})\right)\right)
\end{aligned}
$$

\section{declare slice $I_{i}$ visited.}

\section{end do}

end do

The minimization of the local energy function (4) is conducted by a deterministic optimization algorithm, known as iterated conditional modes (ICM) [12], rendering our method a variant of the ICM. ICM is a discrete Gauss-Seidel-like optimization technique, accepting only configurations decreasing the objective function. Let us notice that the parameter $\hat{\Theta}_{\boldsymbol{i}}$ corresponding to the minimum value of the local energy function $E_{i}\left(\boldsymbol{\Theta}_{\boldsymbol{i}}\right)$ also corresponds to a local minimum value of the global energy function $E(\boldsymbol{\Theta})$ with respect to $\boldsymbol{\Theta}_{\boldsymbol{i}}$, keeping all other parameters $\Theta_{j}, j \neq i$ fixed. Thus, it is easy to see that the described algorithm converges toward a local minimum of the initial energy function (2). This local minimum corresponds to a satisfactory registration, since the initial alignment of the 2-D sections is generally close to the desired solution. If this is not the case, a good initialization may be obtained by a standard coarse alignment technique such as principal axes registration. Thus, it is not necessary to resort here to greedy global optimization procedures, such as simulated annealing or genetic algorithms. Further improvement of the solution is obtained by a gradient descent technique.

The pixel similarity metric associated with the above described global energy function is based on a distance transform [11], [13] (also known as chamfer matching technique [14]) and is computed from the 2-D object contours [15]. A distance transformation is an operation that converts a binary picture, consisting of feature and nonfeature elements (contours), to a picture where each pixel has a value that approximates its distance to the nearest contour point. Thus, using the distance transform $D(\boldsymbol{p})$ of the reference slice the method aligns the floating slice by minimizing the distance between the contours of the images. For further details of the chamfer matching method the reader may refer to [14]. Considering the slices per triplets, which is very common for standard reconstruction problems [i.e., setting $R=1$ in (5)], the estimation of the 
alignment parameters $\Theta$ involves the nonlinear similarity metric

$$
\begin{aligned}
& g\left(\boldsymbol{T}_{\Theta_{\boldsymbol{i}}}(\boldsymbol{p})\right)=D_{i-1}\left(\boldsymbol{T}_{\Theta_{\boldsymbol{i}_{-1}}}(\boldsymbol{p})\right)+D_{i+1}\left(\boldsymbol{T}_{\Theta_{\boldsymbol{i}_{+1}}}(\boldsymbol{p})\right) \\
& I_{i}\left(\boldsymbol{T}_{\boldsymbol{\Theta}_{\boldsymbol{i}}}(\boldsymbol{p})\right) \neq 0
\end{aligned}
$$

where $I_{i}\left(\mathbf{T}_{\Theta_{\mathbf{i}}}(\mathbf{p})\right) \neq 0$ means that only the contour points of $I_{i}$ are involved.

A large number of interpolations are involved in the alignment process. The accuracy of estimation of the rotation and translation parameters is directly related to the accuracy of the underlying interpolation model. Simple approaches such as the nearest neighbor interpolation are commonly used because they are fast and simple to implement, though they produce images with noticeable artifacts. Besides, as the translation and rotation parameters should compensate for accuracy by having subvoxel values, this type of interpolation would not be appropriate. More satisfactory results can be obtained by small-kernel cubic convolution techniques, bilinear, or convolution-based interpolation. According to sampling theory, optimal results are obtained using sinus cardinal interpolation, at the expense of a high computational cost. As a compromise, a bilinear interpolation technique has been used in the optimization procedure. At the end of the algorithm, the alignment parameters are refined using a sinus cardial interpolation that preserves the quality of the image to be aligned. This technique has proven to be fast and efficient.

\section{EXPERIMENTAL RESULTS}

To evaluate our method, we applied the algorithm to the reconstruction of an artificially misaligned 3-D CT scanned mechanical part Fig. 1 . The slices of the original $256 \times 256 \times 109$ CT volume were transformed using translations varying from -10 to +10 pixels and rotations varying from $-20^{\circ}$ to $+20^{\circ}$. The transformations for each slice were random following a uniform distribution in order not to privilege any slice (Fig. 1(a) and (b)). Table I presents statistics on the alignment errors. The algorithm was proven to be robust in aligning this type of image producing small registration errors. Fig. 1(c) and (d) presents the reconstructed volume.

Moreover, we have uniformly transformed 100 slices of the same 3-D volume (mechanical part of an engine) by applying to each slice $I_{i}$ a translation of $t_{x}^{i}=t_{x}^{i-1}+0.2$ pixels and $t_{y}^{i}=t_{y}^{i-1}+0.2$ pixels and a rotation of $\theta^{i}=\theta^{i-1}+0.4^{\circ}$. As the volume has 100 slices, the last slice is translated by 20 pixels in both directions and rotated by $40^{\circ}$ with respect to its initial position. Table II presents the registration errors of the method. It is illustrated that our approach has subpixel mean, median, and maximum errors.

The same evaluation procedure was performed on a 3-D human skull volume with 140 slices (Fig. 2). The algorithm aligned the artificially (randomly and uniformly) misaligned slices of the volume and the errors are drawn in Tables III and IV. Human skull presents discontinuities and consecutive slices may differ significantly due to anatomy, but the global energy function is robust to these shortcomings. As can be seen, median and mean translation and rotation errors are less

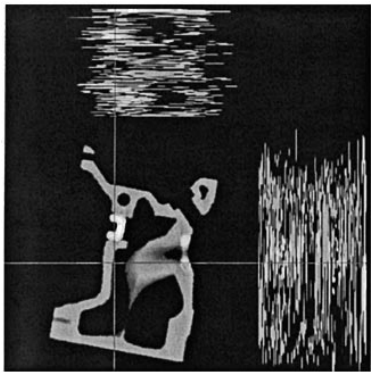

(a)

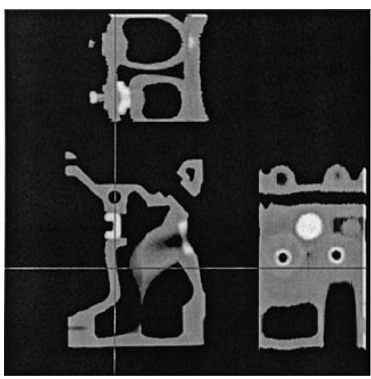

(c)

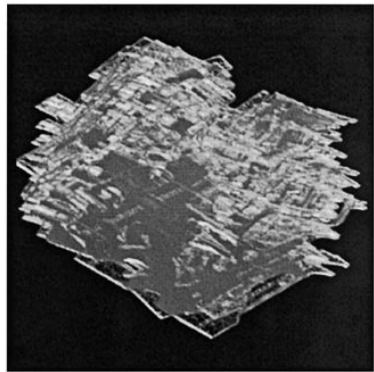

(b)



(d)
Fig. 1. Reconstruction of a 3-D scanned mechanical part volume of 109 slices. (a) Multiplanar view of the volume before registration. (b) 3-D view of the volume before registration. (c) Multiplanar view of the volumeafter registration. (d) 3-D view of the same volume after registration.

TABLE I

SET of 109 SLICES of A 3-D CT SCANNEd Mechanical Part Volume WERE ARTIFICIALLY TRANSFORMED USING DIFFERENT RIGID TRANSFORMATION PARAMETERS. EACH SLICE WAS RANDOMLY Transformed USING TRANSLATIONS VARYING From -10 TO +10 PIXELS AND Rotations VARYING FrOM -20 TO + 20 DEGREES. STATISTICS ON THE AlignMENT ERRORS FOR THE Rigid TRANSFORMATION PARAMETERS ARE Presented. Translation ERrors ARE EXPRESSED IN PIXELS AND ROTATION ERROR IN DEGREES

\begin{tabular}{l|c|c|c}
\hline & $\Delta t_{x}$ & $\Delta t_{y}$ & $\Delta \theta$ \\
\hline \hline median & 0.33 & 0.31 & 0.06 \\
\hline maximum & 1.07 & 0.93 & 0.25 \\
\hline mean $\pm \mathrm{s.dev}$ & $0.35 \pm 0.25$ & $0.38 \pm 0.25$ & $0.07 \pm 0.06$ \\
\hline
\end{tabular}

TABLE II

SET of 100 Slices of a 3-D CT Scanned Mechanical Part Volume WERE ARTIFICIALLY TRANSFORMED USING DIFFERENT RIGID Transformation Parameters. EACH Slice Was Translated By 0.2 PIXELS IN BOTH DIRECTIONS AND ROTATED BY 0.4 DEGREES WITH RESPECT TO ITS PRECEDING SLICE. DifFERENT Statistics ON THE ERRORS FOR THE Rigid Transformation PARAMETERS ARE PRESENTED. TRANSLATION ERRORS ARE EXPRESSED IN PIXELS AND ROTATION ERROR IN DEGREES

\begin{tabular}{l|c|c|c}
\hline & $\Delta t_{x}$ & $\Delta t_{y}$ & $\Delta \theta$ \\
\hline \hline median & 0.19 & 0.23 & 0.05 \\
\hline maximum & 0.99 & 0.87 & 0.32 \\
\hline mean \pm s. dev & $0.29 \pm 0.26$ & $0.31 \pm 0.26$ & $0.07 \pm 0.07$ \\
\hline
\end{tabular}

than 1 pixel and 1 degree, respectively. Also maximum errors are slightly larger than one pixel and one degree, respectively, showing the robustness of the proposed technique.

Furthermore, the algorithm was applied to the reconstruction of volumes (tooth germs, biological tissues) with unknown 




(a)

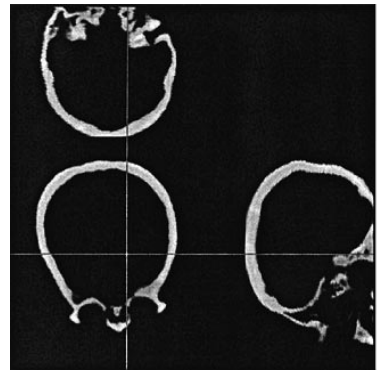

(c)

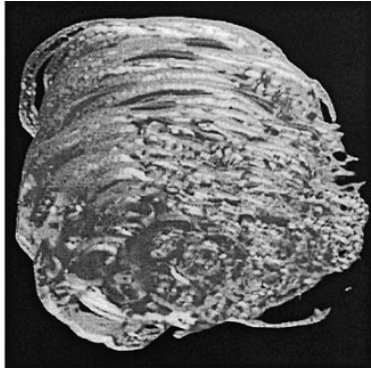

(b)

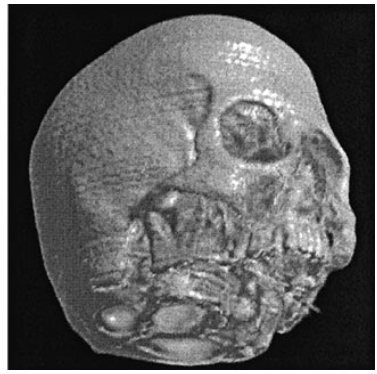

(d)
Fig. 2. Reconstruction of a 3-D human skull volume of 140 slices. (a) Multiplanar view of the volume before registration. (b) 3-D view of the volume before registration. (c) Multiplanar view of the volume after registration. (d) 3-D view of the same volume after registration.

TABLE III

SET of 140 Slices of a 3-D CT Human SKull Volume Were ARTificially TRANSFORMED USING DIFFERENT RIGID TRANSFORMATION PARAMETERS. EACH SLICE WAS RANDOMLY TRANSFORMED USING TRANSLATIONS VARYING

FROM -10 TO + 10 PIXELS AND ROTATIONS VARYING FROM -20 TO +20 DEGREES. DIFFERENT STATISTICS ON THE ERRORS FOR THE RIGID Transformation Parameters ARE Presented. Translation ERrors ARE EXPRESSED IN PIXELS AND ROTATION ERROR IN DEGREES

\begin{tabular}{l|c|c|c}
\hline & $\Delta t_{x}$ & $\Delta t_{y}$ & $\Delta \theta$ \\
\hline \hline median & 2.10 & 0.33 & 0.07 \\
\hline maximum & 1.45 & 2.02 & 2.42 \\
\hline mean \pm s. dev & $0.37 \pm 0.28$ & $0.38 \pm 0.30$ & $0.19 \pm 0.35$ \\
\hline
\end{tabular}

ground truth. The performance of our method was compared with the manual alignment accomplished by an expert physician-researcher. Fig. 3 shows the reconstruction of a tooth germ (acquired using an optical microscopy) by an expert dentist-researcher [Fig. 3(c) and (d)] and by our method [Fig. 3(e) and (f)]. It is illustrated that human intervention fails to correctly align the slices, while our method is efficient and can achieve alignment with high accuracy, which has been confirmed by dentist specialists. The same stands for the example presented in Fig. 4 where another tooth reconstruction is presented. In both examples, a specialist (dentist-researcher) confirmed that the volumes reconstructed by our method are of higher quality. This can also be observed by a simple visual inspection (Figs. 3 and 4).

In both examples, the visual quality of the teeth under consideration are superior regarding the smoothness and the curvature of their surfaces aligned by our program, according to the validation performed by an objective (unbiased) dentist-researcher, who was knowledgeable of the teeth in their real form.

Moreover, we applied the algorithm to the reconstruction of an already aligned 3-D human skull volume (the ground truth

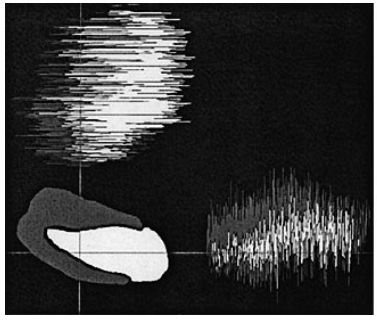

(a)



(c)

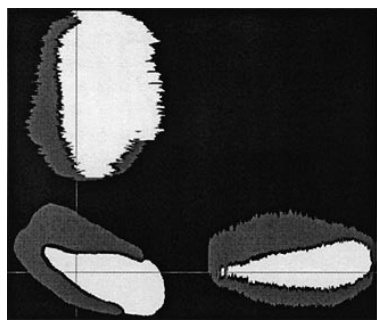

(e)

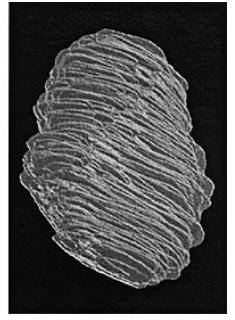

(b)

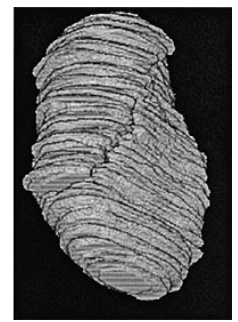

(d)



(f)
Fig. 3. Reconstruction of a 3-D tooth germ volume of 265 slices. (a) Multiplanar view of the volume before registration. (b) 3-D view of the volume before registration. (c) Multiplanar view of the volume after manual alignment by an expert dentist-researcher. (d) 3-D view of the volume after manual alignment by an expert dentist-researcher. (e) Multiplanar view of the volume after registration. (f) 3-D view of the same volume after registration.

of Fig. 2). The results shown in Table $\mathrm{V}$ illustrate that our algorithm is unbiased. As it can be seen, median and mean translation and rotation errors are less that 0.2 pixel and $0.1^{\circ}$, respectively. Maximum errors are also about 1 pixel and less than $0.5^{\circ}$, respectively, proving the unbiasness of our method.

Finally, let us notice that the algorithm has a computational complexity $O\left(N_{x} N_{y} N\right)$ and requires approximately $10 \mathrm{~min}$, to reconstruct a $256 \times 256 \times 140$ volume on a Pentium III $(800 \mathrm{MHz})$ workstation under Windows 2000 Professional without any particular code optimization.

\section{CONCLUSION}

The alignment method described in this paper is akin to the global energy function formulation proposed in [9] to register multiple views of a 3-D surface in computer vision applications. The main contribution of our approach is to consider the alignment problem globally on the 3-D volume, by minimizing a global objective function expressing the similarity between neighboring slices. The approach does not privilege any particular direction in the registration process. By these means, the major problems set by the registration of serially acquired slices are addressed. With the global (isotropic) formulation of the registration problem (rather than a standard step by step, sequential formulation), no global offset nor error propagations 


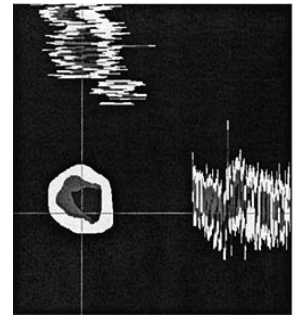

(a)

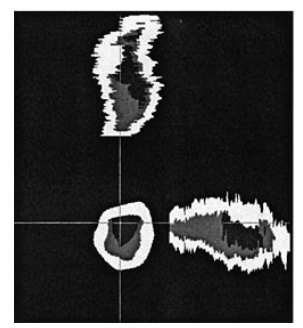

(c)

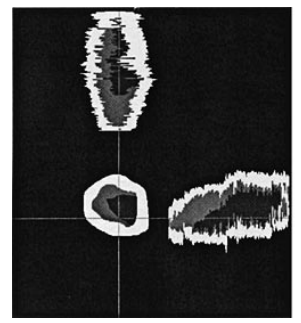

(e)

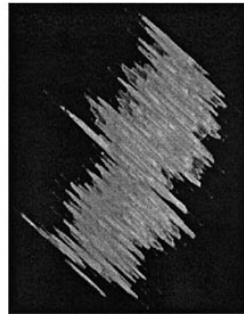

(b)



(d)

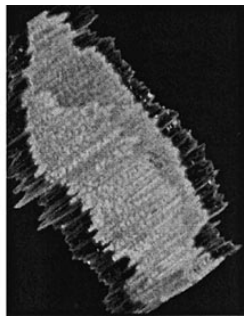

(f)
Fig. 4. Reconstruction of a 3-D tooth germ volume of 194 slices. (a) Multiplanar view of the volume before registration. (b) 3-D view of the volume before registration. (c) Multiplanar view of the volume after manual alignment by an expert dentist-researcher. (d) 3-D view of the volume after manual alignment by an expert dentist-researcher. (e) Multiplanar view of the same volume after registration. (f) 3-D view of the same volume after registration.

TABLE IV

SET of 140 Slices of A 3-D CT HuMAN SKUll Volume Were ARTIFICIALly TRANSFORMED USING DIFFERENT RIGID TRANSFORMATION PARAMETERS.

EACH SLICE Was TRANSLATED By 0.15 PIXELS IN Both DiRECTIONS AND Rotated By 0.3 Degrees With Respect to ITS PRECEDING SLICE.

DIFFERENT STATISTICS ON THE ERRORS FOR THE RIGID TRANSFORMATION Parameters Are Presented. TRANSLATION ERRORS ARE EXPressed IN PiXEls AND Rotation ERROR IN DEGREES

\begin{tabular}{l|c|c|c}
\hline & $\Delta t_{x}$ & $\Delta t_{y}$ & $\Delta \theta$ \\
\hline \hline median & 0.23 & 0.21 & 0.26 \\
\hline maximum & 1.95 & 1.94 & 1.64 \\
\hline mean $\pm \mathrm{s.dev}$ & $0.33 \pm 0.32$ & $0.34 \pm 0.33$ & $0.25 \pm 0.25$ \\
\hline
\end{tabular}

TABLE V

AlREAdy Aligned Set of 140 SLICES of A 3-D CT Human SKULl Volume (The Ground Truth) Were APPliEd to the Algorithm. Translation ERRORS ARE EXPRESSED IN PIXELS AND ROTATION ERROR IN DEGREES

\begin{tabular}{l|c|c|c}
\hline & $\Delta t_{x}$ & $\Delta t_{y}$ & $\Delta \theta$ \\
\hline \hline median & 0.012 & 0.017 & 0.004 \\
\hline maximum & 0.980 & 1.001 & 0.492 \\
\hline mean $\pm \mathrm{s} . \operatorname{dev}$ & $0.082 \pm 0.159$ & $0.165 \pm 0.281$ & $0.032 \pm 0.092$ \\
\hline
\end{tabular}

are observed in the final alignment. Its association to more sophisticated but time consuming pixel similarity metrics (mutual information [16], robust estimation-based measures [17]) may improve its accuracy and is a perspective of this work.

\section{ACKNOWLEDGMENT}

The authors wish to thank Prof. K. Lyroudia, Department of Dentistry, Aristotle University of Thessaloniki, for providing the dental images and evaluating the registration results, as well as A. Digka, dentist and Ph.D. student in endodontology in the Department of Dentistry, for providing the pulp tissue's blood vessels volume.

\section{REFERENCES}

[1] J. B. A. Maintz and M. A. Viergever, "A survey of medical image registration techniques," Med. Image Anal., vol. 2, no. 1, pp. 1-36, 1998.

[2] P. Van den Elsen, E. J. D. Paul, and M. A. Viergever, "Medical image matching-A review with classification," IEEE Eng. Med. Biol. Mag., vol. 12, pp. 26-39, 1993.

[3] A. F. Goldszal, O. J. Tretiak, P. J. Hand, S. Bhasin, and D. L. Mac Eachron, "Three-dimensional reconstruction of activated columns from $2-\left[{ }^{14} \mathrm{C}\right]$ deoxy-D-glucose data," NeuroImage, vol. 2, pp. 9-20, 1995.

[4] E. Bardinet, L. D. Cohen, and N. Ayache, "Analyzing the deformation of the left ventricle of the heart with a parametric deformable model," INRIA Sophia Antipolis, France, Tech. Rep. RR-2797, 1996.

[5] A. Rangarajan, H. Chui, E. Mjolsness, S. Pappu, L. Davachi, P. Goldman-Rakic, and J. Duncan, "A robust point-matching algorithm for autoradiograph alignment," Med. Image Anal., vol. 1, no. 4, pp. 379-398, 1997.

[6] A. Andreasen, A. M. Drewes, J. E. Assentoft, and N. E. Larsen, "Computer-assisted alignment of standard serial sections without use of artificial landmarks. A practical approach to the utilization of incomplete information of 3-D reconstruction of the hippocampal region," J. Neurosci. Methods, vol. 45, pp. 199-207, 1992.

[7] B. Kim, J. Boes, K. Frey, and C. Meyer, "Mutual information for automated unwarping of rat brain autoradiographs," NeuroImage, vol. 5, pp. 31-40, 1997.

[8] S. Ourselin, A. Roche, G. Subsol, X. Pennec, and C. Sattonnet, "Automatic alignment of histological sections for 3-D reconstruction and analysis," Ecole National de Rechearche en informatique et en automatique, Tech. Rep. 3595, 1998.

[9] R. Ben-Jemaa and F. Schmitt, "A solution for the registration of multiple 3-D points sets using unit quaternions," in Proc. 5th Eur. Conf. Computer Vision (ECCV'98), vol. 2, Freiburg, Germany, June 1998, pp. 34-50.

[10] M. J. Besl and N. McKay, "A method for the registration of 3-D shapes," IEEE Trans. Pattern Anal. Machine Intell., vol. 14, pp. 239-256, Feb. 1992.

[11] G. Borgefors, "Distance transformations in arbitrary dimensions," Comput. Vision, Graphics Image Processing, vol. 27, pp. 321-345, 1984.

[12] J. Besag, "On the statistical analysis of dirty pictures," J. Roy. Statist. Soc., vol. 48, no. 3, pp. 259-302, 1986.

[13] P.-E. Danielsson, "Euclidean distance transform," Comput. Graphics Image Processing, vol. 14, pp. 227-228, 1980.

[14] G. Borgefors, "Hierarchical chamfer matching: A parametric edge matching algorithm," IEEE Trans. Pattern Anal. Machine Intell., vol. 10, pp. 849-965, Nov. 1988.

[15] J. Canny, "A computational approach to edge detection," IEEE Trans. Pattern Anal. Machine Intell., vol. PAMI-8, pp. 679-698, Nov. 1986.

[16] W. Wells III, P. Viola, H. Atsumi, S. Nakajima, and R. Kikinis, "Multimodal volume registration by maximization of mutual information," Med. Image Anal., vol. 1, no. 1, pp. 33-51, 1996.

[17] C. Nikou, J. P. Armspach, F. Heitz, I. J. Namer, and D. Grucker, "MR/MR and MR/SPECT registration of brain images by fast stochastic optimization of robust voxel similarity measures," NeuroImage, vol. 8, no. 1, pp. 30-43, 1998. 
Stelios Krinidis was born in Kavala, Greece, in 1978. He received the Diploma degree in informatics from the Aristotle University of Thessaloniki, Thessaloniki, Greece, in 1999 and is pursuing the Ph.D. degree in 3-D image processing at the same university.

In June 2000, he joined the Artificial Intelligence and Information Analysis group at the same university, where he is currently a Researcher and Teaching Assistant. His research interests include 3-D image processing and analysis and computer vision.

Christophoros Nikou (S'97-A'99) was born in Thessaloniki, Greece, in 1971 $\mathrm{He}$ received the $\mathrm{Ph} . \mathrm{D}$. degree in image processing and computer vision in 1999, the DEA degree in optoelectronics and image processing in 1995, both from Louis Pasteur University, Strasbourg, France, and the Diploma degree in electrical engineering from the Aristotle University of Thessaloniki in 1994.

During 2001, he was a Senior Researcher with the Department of Informatics, Aristotle University of Thessaloniki, where he conducted research in image processing in the framework of various European projects. Since 2002, he has been with Compucon S.A., Thessaloniki, Greece, managing research projects in 3-D medical image processing and analysis. His research interests mainly include computer vision, pattern recognition, biomedical image processing, image registration and segmentation, deformable models, statistical image processing.

Dr. Nikou is a member of the Technical Chamber of Greece.
Ioannis Pitas (SM'94) received the Diploma degree in electrical engineering in 1980 and the Ph.D. degree in electrical engineering in 1985, both from the University of Thessaloniki, Thessaloniki, Greece.

From 1980 to 1993, he served as Scientific Assistant, Lecturer, Assistant Professor, and Associate Professor with the Department of Electrical and Computer Engineering at the Department of Informatics, University of Thessaloniki. Since 1994, he has been a Professor at the same university. He served as a Visiting Research Associate at the University of Toronto, Toronto, ON, Canada, University of Erlangen-Nuernberg, Germany, Tampere University of Technology, Tampere, Finland, as Visiting Assistant Professor at the University of Toronto and as Visiting Professor at the University of British Columbia, Vancouver, Canada. He was Lecturer in short courses for continuing education. His current interests are in the areas of digital image processing, multidimensional signal processing, watermarking, and computer vision. $\mathrm{He}$ has published more than 380 papers and contributed to 13 books in his areas of interest, including Nonlinear Digital Filters: Principles and Applications (Boston, MA: Kluwer, 1990), 3-D Image Processing Algorithms (New York: Wiley, 2000), Nonlinear Model-Based Image/Video Processing and Analysis (New York: Wiley, 2001) and author of Digital Image Processing Algorithms and Applications (New York: Wiley, 2000). He is the editor of the book Parallel Algorithms and Architectures for Digital Image Processing, Computer Vision and Neural Networks (New York: Wiley, 1993).

Dr. Pitas has been a Member of the European Community ESPRIT Parallel Action Committee. He has also been an Invited Speaker and/or Member of the program committee of several scientific conferences and workshops. He was Associate Editor of the IEEE TRANSACTIONS ON CIRCUITS AND SYSTEMS, Associate Editor of the IEEE TRANSACTIONS ON NEURAL NETWORKS, Co-Editor of Multidimensional Systems and Signal Processing and he is currently an Associate Editor of the IEEE TRANSACTIONS ON IMAGE PROCESSING. He was General Chair of the 1995 IEEE Workshop on Nonlinear Signal and Image Processing (NSIP95), Technical Chair of the 1998 European Signal Processing Conference, and General Chair of IEEE ICIP2001. 